\title{
Profile of guanfacine extended release and its potential in the treatment of attention-deficit hyperactivity disorder
}

This article was published in the following Dove Press journal:

Neuropsychiatric Disease and Treatment

28 May 2015

Number of times this article has been viewed

\author{
Jose Martinez-Raga ${ }^{1,2}$ \\ Carlos Knecht ${ }^{3}$ \\ Raquel de Alvaro ${ }^{4}$ \\ 'Teaching Unit of Psychiatry and \\ Psychological Medicine, University \\ Hospital Doctor Peset, University \\ of Valencia, ${ }^{2}$ CEU Cardenal Herrera \\ University, ${ }^{3}$ Área de Salud Mental, \\ Hospital Padre Jofré, Valencia, ${ }^{4}$ Hospital \\ General, Consorcio Hospitalario \\ Provincial, Castellon, Spain
}

\begin{abstract}
The $\alpha_{2}$-adrenergic receptor agonist guanfacine, in its extended-release formulation (GXR), is the most recent nonstimulant medication approved in several countries for the treatment of attention-deficit hyperactivity disorder (ADHD) as monotherapy and as adjunctive pharmacotherapy to stimulants in children and adolescents. The present paper aims to review comprehensively and critically the pharmacodynamic and pharmacokinetic characteristics and the published evidence on the efficacy and safety profile of GXR in the treatment of ADHD. A comprehensive search of relevant databases (PubMed, Embase, and PsycInfo) was conducted to identify studies published in peer-reviewed journals until January 15, 2015. Though the precise mechanism of action of guanfacine in the treatment of ADHD is not fully understood, it is thought to act directly by enhancing noradrenaline functioning via $\alpha_{2 \mathrm{~A}}$-adrenoceptors in the prefrontal cortex. Weight-adjusted doses should be used, with a dosing regime on a milligram per kilogram basis, starting at doses in the range $0.05-0.08 \mathrm{mg} / \mathrm{kg} / \mathrm{day}$, up to $0.12 \mathrm{mg} /$ $\mathrm{kg} /$ day. As evidenced in short-term randomized controlled trials and in long-term open-label extension studies, GXR has been shown to be effective as monotherapy in the treatment of ADHD. Furthermore, GXR has also been found to be effective as adjunctive therapy to stimulant medications in patients with suboptimal responses to stimulants. Many of the adverse reactions associated with GXR, particularly sedation-related effects, were dose-related, transient, mild to moderate in severity, and did not interfere with attention or overall efficacy. There are no reports of serious cardiovascular adverse events associated with GXR alone or in combination with psychostimulants.
\end{abstract}

Keywords: guanfacine extended-release, attention-deficit hyperactivity disorder, ADHD, oppositional symptoms, adjunctive therapy, cardiovascular safety

\section{Introduction}

Attention-deficit hyperactivity disorder (ADHD) is a complex, heterogeneous, and highly prevalent neurodevelopmental disorder characterized by a persistent pattern of age-inappropriate symptoms of inattention, hyperactivity, and/or impulsivity. ${ }^{1,2}$ The prevalence of ADHD is approximately 5\%-8\% in children and $2.5 \%-4 \%$ in adults worldwide. ${ }^{1,3,4}$ Along the life span, ADHD is associated with important personal, medical, and social complications, significant functional impairment, and high rates of associated psychiatric disorders. ${ }^{1,5-7}$ Conduct disorders, including oppositional defiant disorder (ODD), anxiety disorders, and mood disorders, are the most common psychiatric comorbidities in children and adolescents with ADHD. 1,5,8,9

ADHD has multifactorial origins, resulting from the complex interaction of biological and environmental risk factors, including multiple genetic factors in conjunction with prenatal events and infant complications. ${ }^{10,11}$ A growing body of evidence reveals 
ADHD-related dysfunctions in multiple neuronal networks involved in higher-level cognitive and sensorimotor functions. ${ }^{12}$ However, the frontostriatal network, which involves structures like the lateral prefrontal cortex (PFC), dorsal anterior cingulate cortex, caudate nucleus, and putamen, linked with attention, impulsivity, and activity control, appears to be key in understanding the pathophysiology of ADHD. ${ }^{13,14}$

Since the publication in 1937 of a study showing the therapeutic effects of Benzedrine, the racemic mixture of levo- and dextroamphetamine for hyperactive, inattentive children, ${ }^{15}$ stimulants, including amphetamine (AMP) derivatives and methylphenidate $(\mathrm{MPH})$ formulations, have been widely use and remain first-line pharmacotherapies for individuals with ADHD, due to their evidence-based efficacy. ${ }^{9,16-18}$ However, a proportion of patients do not tolerate or fail to respond adequately to stimulants. ${ }^{19,20}$ In addition, some patients or their caregivers prefer not to take stimulant medications, and in some cases there are contraindications to MPH- or AMP-based treatments, including symptomatic cardiovascular disease, glaucoma, or some severe or unstable comorbid psychiatric disorders, as well as concerns about drug diversion., ${ }^{9,21,22}$ Consequently, other safe and effective pharmacotherapies have emerged for treating patients with ADHD. Atomoxetine (ATX; Strattera ${ }^{\circledR}$; Eli Lilly and Company, Indianapolis, IN, USA), ${ }^{23}$ a selective noradrenalinereuptake inhibitor, was the first nonstimulant drug approved for the treatment of ADHD, and in Europe is at present the only medication approved to be used in adult patients with ADHD. More recently, following the results of pivotal, randomized, double-blind, placebo-controlled studies ${ }^{24-26}$ showing the efficacy and good tolerability profile of guanfacine in its extended-release formulation (GXR) in youth diagnosed of ADHD, this $\alpha_{2}$-adrenergic receptor agonist has been approved (Intuniv ${ }^{\circledR}$; Shire PLC, Wayne, Dublin, Ireland) in several countries for the treatment of ADHD as monotherapy and as adjunctive therapy to stimulant medications in children and adolescents (6-17 years of age).

Although some recent reports, ${ }^{22,27}$ including a systematic review and meta-analysis, ${ }^{28}$ have reviewed the usefulness of GXR in the treatment of ADHD, additional evidence has been published on the efficacy, tolerability, and cost-effectiveness of this $\alpha_{2}$-adrenergic receptor agonist as a pharmacotherapy for ADHD. Therefore, the present paper aims to provide a comprehensive, critical, and updated review on the pharmacodynamic and pharmacokinetic characteristics and published evidence on the efficacy, cost-effectiveness, and safety and tolerability profile of GXR in the treatment - as monotherapy or as an add-on medication - of children and adolescents with ADHD.

\section{Materials and methods}

A comprehensive search of relevant databases (PubMed, Embase, and PsycInfo) was conducted to identify potentially relevant studies published in English in peer-reviewed journals until January 15, 2015, using the following search terms: ("guanfacine" or "guanfacine extended-release") and ("child" or "adolescent" or "pediatrics") and ("ADHD" or "attention deficit disorder" or "attention hyperactivity disorder" or "hyperactivity" or "hyperkinetic disorder"). The literature search included clinical studies with children or adolescents aged 6-17 years as participants. There were no restrictions on the identification or inclusion of studies in terms of publication status, type of publication, or design type. However, abstracts of presentations to specialist meetings and conferences were not included. Titles and abstracts were screened for inclusion/exclusion and appropriateness, and full-text versions were retrieved.

\section{Pharmacology of guanfacine}

Guanfacine is an agonist at $\alpha_{2 \mathrm{~A}}$-adrenergic receptor distributed in different brain regions, but heavily concentrated in the PFC and the locus coeruleus. In addition to their primary indication as antihypertensive agents, $\alpha_{2}$-adrenergic receptor agonists have been used for a variety of childhood and adult psychiatric disorders, including migraine, Tourette's syndrome, nicotine dependence, and opiate-withdrawal symptoms, as well as ADHD. ${ }^{27,29-32}$ Guanfacine's beneficial actions in ADHD are likely to be mediated by its ability to potentiate noradrenaline neurotransmission and strengthen PFC network connections for the regulation of attention, emotion, impulsivity, and behavior through actions at postsynaptic $\alpha_{2}$ receptors. ${ }^{33}$

\section{Pharmacodynamic profile of guanfacine}

Guanfacine is a selective $\alpha_{2 \mathrm{~A}}$-adrenergic receptor agonist that shares some pharmacological properties with the nonselective $\alpha_{2}$-agonist clonidine, which in an extended-release formulation (Kapvay ${ }^{\circledR}$; Concordia Pharmaceuticals Inc, Bridgetown, Barbados) has also been approved by the US Food and Drug Administration for the treatment of ADHD in children and adolescents as monotherapy or as adjunctive therapy to stimulants. ${ }^{34}$ However, while clonidine shows high affinity for all three subtypes of $\alpha_{2}$-receptors (A, B, and C), as well as for imidazoline receptors, guanfacine appears to bind more preferentially to postsynaptic $\alpha_{2 \mathrm{~A}}$-adrenergic receptors, as it has been reported to have 15-20 timeshigher affinity for $\alpha_{2 \mathrm{~A}}$-adrenergic receptors than for the $\alpha_{2 \mathrm{~B}}$ or $\alpha_{2 \mathrm{C}}$ subtypes. ${ }^{32,35}$ These differences in receptor affinities between both $\alpha_{2}$-adrenergic agonists may help to explain why 
guanfacine causes less sedation and dizziness, and is not as potent in decreasing blood pressure (BP) as clonidine. ${ }^{36}$

Although the precise mechanism of action of guanfacine in the treatment of ADHD is not fully understood, this $\alpha_{2}$-agonist, which has no central nervous system-stimulant properties, is thought to act directly by enhancing noradrenaline functioning via $\alpha_{2 \mathrm{~A}}$-adrenoceptors in the PFC. ${ }^{37,38}$ There are presynaptic and postsynaptic $\alpha_{2 \mathrm{~A}}$-adrenergic receptors, but guanfacine appears to act primarily at postsynaptic receptors through inhibition of cyclic adenosine monophosphate, closing hyperpolarization-activated cyclic nucleotide-gated channels modulating synaptic functioning and strengthening PFC-network connectivity, thereby improving PFC cognitive functions. ${ }^{37,39}$ As a result, guanfacine is hypothesized to enhance the signal-to-noise ratio from environmental stimuli, and may improve the ability to focus on a particular stimulus "above the noise" during periods of low arousal, thus improving symptoms associated with ADHD, including working memory, planning, organization, and response inhibition. ${ }^{14,35,39}$

\section{Pharmacokinetics of guanfacine}

GXR tablets contain guanfacine hydrochloride in a matrix tablet formulation that includes polymers and organic acids to provide extended release of the active drug throughout the gastrointestinal tract. ${ }^{33,40}$ GXR is readily absorbed following oral administration, and has linear pharmacokinetics after administration of single $2 \mathrm{mg}$ doses and repeated $2 \mathrm{mg}$ and $4 \mathrm{mg}$ doses in children and adolescents with ADHD. ${ }^{27,40,41}$ While immediate-release guanfacine (GIR) has an oral bioavailability of approximately $80 \%,{ }^{42}$ the bioavailability of GXR is $58 \% .^{22,33}$ Furthermore, compared with GIR, GXR was associated with a $60 \%$ reduction in peak plasma concentration $\left(\mathrm{C}_{\max }\right)$ and a $43 \%$ reduction in area under the plasma concentration-time curve (AUC). ${ }^{27,33}$ Conversely, GXR exhibits a time to $\mathrm{C}_{\text {max }}$ twice as long as GIR, reaching a time to $\mathrm{C}_{\max } 6$ hours after GXR dosing compared with 3 hours after dosing with GIR. ${ }^{41,43}$

The pharmacokinetics of GXR do not seem to change with dose, but are significantly affected by food intake. When administered with a high-fat meal, exposure to guanfacine from GXR is increased significantly, with approximately a $40 \%$ increase in AUC and a $75 \%$ increase in $\mathrm{C}_{\max }$ compared to dosing in a fasted state. ${ }^{33,40,41}$ The food effect could be clinically significant, because many of the important adverse effects of GXR occurred in a dose-related or exposure-related manner. Therefore, in order to minimize the risk of adverse events (AEs), GXR should be administered with a light meal or without food. ${ }^{33}$ Guanfacine is moderately bound to plasma protein, with $64 \%-72 \%$ bound to plasma proteins, independent of drug concentration. ${ }^{42}$

Guanfacine is cleared both by the liver and the kidney. GXR has a long elimination half-life: 14.4 hours in children and 17.9 hours adolescents after a single $2 \mathrm{mg}$ dose. ${ }^{40}$ Guanfacine is metabolized by oxidation to form 3-hydroxyguanfacine, its main metabolite. ${ }^{42}$ As evidenced in an in vitro study assessing P-glycoprotein substrate properties, it appears that the role of P-glycoprotein in guanfacine transport is at best minor and unlikely to be of clinical relevance in interindividual variability in response to guanfacine therapy. ${ }^{44}$ The cytochrome P450 (CYP)-3A4 isoenzyme is the primary enzyme involved in the oxidative metabolism of guanfacine. ${ }^{33}$ However, while it does not appear to be an inhibitor of the major CYP isoenzymes, guanfacine is a substrate of CYP3A4/5. ${ }^{33}$ Concomitant administration with drugs that inhibit CYP3A4/5 activity significantly increased plasma concentrations of guanfacine, potentially increasing the incidence and severity of AEs. ${ }^{22,45}$ Likewise, administration of guanfacine and another medication that induces the CYP3A4 system, like rifampicin, may decrease the $\mathrm{C}_{\max }$ of guanfacine by more than $50 \% .{ }^{45}$ In addition, when guanfacine and valproic acid are administered simultaneously, plasma valproate levels may rise significantly. ${ }^{46}$ However, two small openlabel studies conducted with healthy adults revealed that coadministration of GXR and $\mathrm{MPH}^{47}$ or lisdexamfetamine ${ }^{48}$ did not result in significant pharmacokinetic drug-drug interactions.

\section{Dosage and administration}

GXR is intended for once-daily dosing. Tablets should be swallowed whole and not be crushed, chewed, or split before swallowing, as this would increase the rate of guanfacine release. ${ }^{33}$ Tablets should not be administered with high-fat meals. ${ }^{41}$ It is recommended that patients commence treatment with a dose of $1 \mathrm{mg}$ per day, thereafter adjusting in weekly increments of no more than $1 \mathrm{mg}$, depending on clinical response and tolerability. ${ }^{24,25,49,50}$ However, weightadjusted doses are preferable, by using a dosing regime on a milligram-per-kilogram basis, considering the results from randomized controlled trials (RCTs), starting at doses in the range of $0.05-0.08 \mathrm{mg} / \mathrm{kg} /$ day, up to $0.12 \mathrm{mg} / \mathrm{kg} /$ day. ${ }^{33,49,51,52}$ Indeed, as reported in an open-label dose-escalation pharmacokinetic evaluation, ${ }^{40}$ with equal doses of GXR, observed guanfacine plasma concentrations were higher in children than in adolescents and adults, presumably due to the lower body weight of children. ${ }^{42}$ Doses above $4 \mathrm{mg}$ /day in children (aged 6-12 years) and above $7 \mathrm{mg} /$ day in adolescents (aged 13-17 years) have not been studied. Likewise, doses above 
$4 \mathrm{mg} /$ day have not been evaluated as adjunctive medication to stimulants. ${ }^{33}$ Treatment discontinuation of GXR should not be done abruptly, but should be tapered in decrements of no greater than $1 \mathrm{mg}$ every 3-7 days, to minimize the risk of transient increases in BP and heart rate (HR) ${ }^{33}$

\section{Efficacy of guanfacine extended release for ADHD}

A systematic review and meta-analysis of published and unpublished studies indicated that compared with placebo, guanfacine was safe and effective in treating children and adolescents with ADHD. ${ }^{28}$ This meta-analysis, which included 1,752 patients of pediatric age from seven RCTs with durations of 6-16 weeks, revealed that overall, 694 of 1,177 (59.0\%) children or adolescents in the guanfacine group benefited from the treatment compared to 192 of 575 (33.3\%) in the placebo group (pooled odds ratio [OR] 3.2).

\section{Efficacy of guanfacine extended release as monotherapy for ADHD}

As summarized in Table 1, several multicenter studies have assessed the efficacy of GXR in the treatment of children and adolescents with ADHD as monotherapy in short-term $\operatorname{RCTs}^{24,25,49,51-53}$ and long-term extension studies, ${ }^{54,55}$ as well as adjunctive pharmacotherapy in combination with stimulants for those patients who failed to have an adequate response with the latter medication. ${ }^{26,50}$ All the different studies but one relied on changes on changes from baseline in the Attention-Deficit/ Hyperactivity Disorder Rating Scale IV (ADHD-RS-IV) as the primary outcome measure, with one exception ${ }^{53}$ that used the ADHD-RS-IV as secondary outcome measure. Other secondary measures in the different reports included the BeforeSchool Functioning Questionnaire, Conners Global Index Parent, Conners Parent Rating Scale - revised: short form (CPRS-R:SF), CPRS-R: long form (CPRS-R:LF), Conners Teacher Rating Scale - revised, Clinical Global Impression improvement (CGI-I), CGI - severity, Parent Global Assessment (PGA), and Child Health Questionnaire - parent form.

\section{Efficacy of GXR for children and adolescents with ADHD in short-term studies}

The efficacy of GXR monotherapy was initially assessed in two similarly designed, pivotal placebo-controlled RCTs in children and adolescents with ADHD (Table 1). ${ }^{24,25}$ In these two short-term trials, patients were assigned to receive either GXR 2, 3, or $4 \mathrm{mg}(\mathrm{n}=345),{ }^{24}$ GXR 1, 2, 3, or $4 \mathrm{mg}$ $(\mathrm{n}=324),{ }^{25}$ or placebo. In either study, all treatment groups

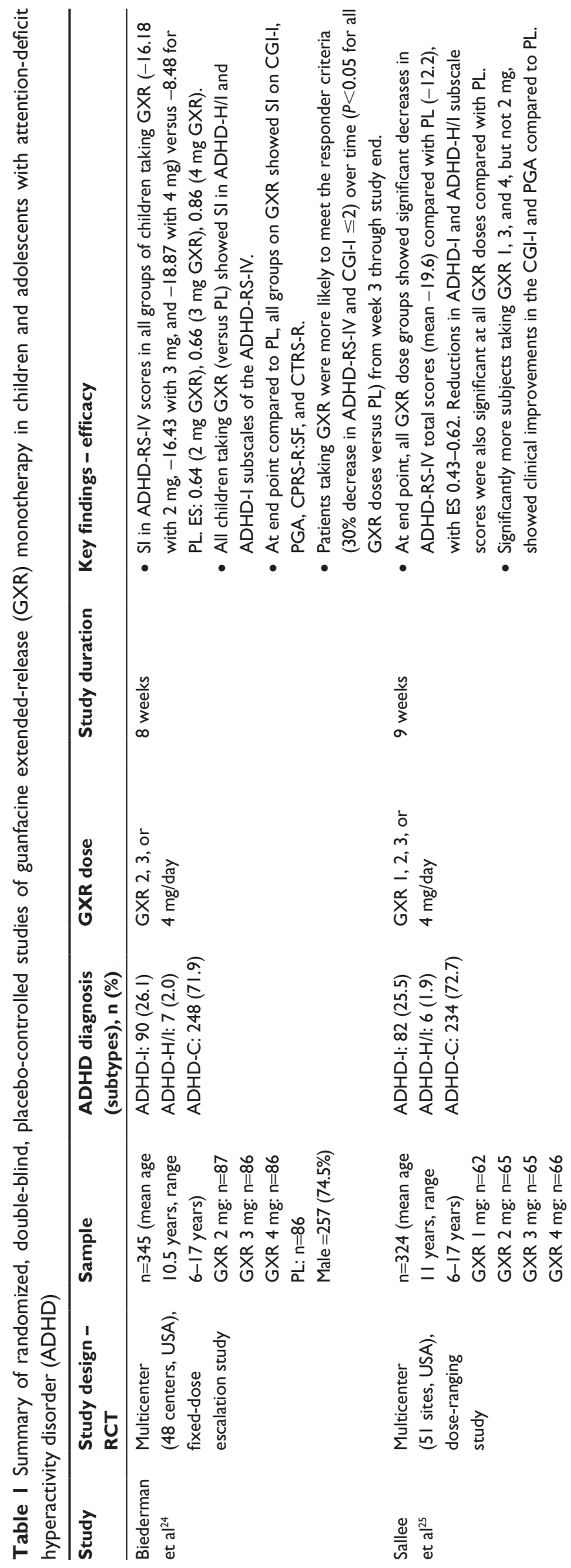



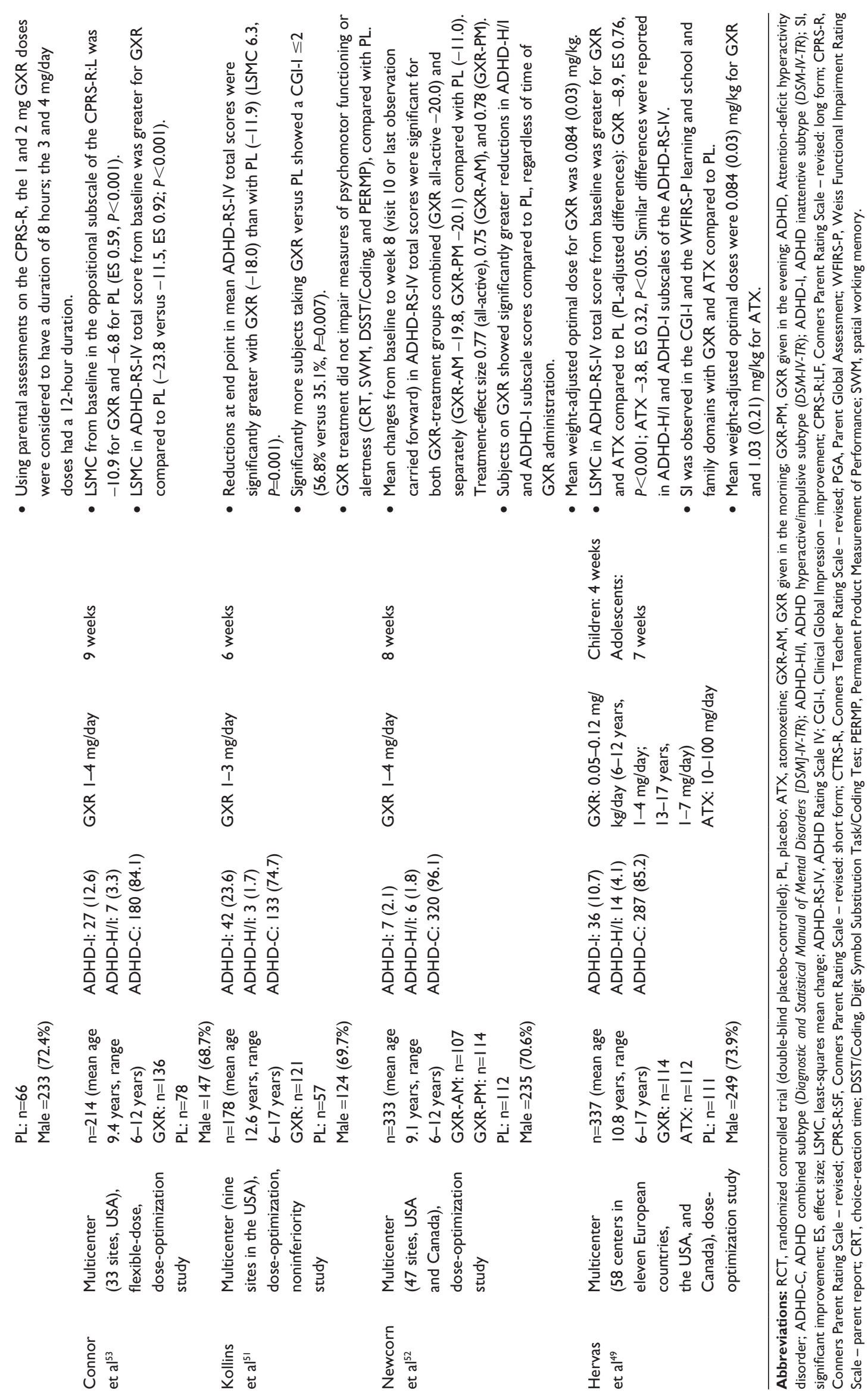

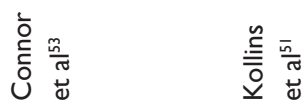

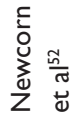

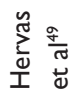


showed significant improvements in ADHD core symptoms, as indicated by significant reductions in ADHD-RS-IV scores at end point compared to placebo, starting 1-2 weeks after they began receiving once-daily GXR, with effect sizes (ESs) ranging from 0.43 to $0.86 .^{24,25}$ Post hoc subgroup analyses revealed that while children 6-12 years of age taking any of the GXR doses in either study exhibited significant improvement compared to placebo, this was not the case for adolescents (aged 13-17 years). Although the small sample size of this cohort in both studies $-23.2 \%(n=80)^{24}$ and $25 \%(n=80)^{25}$ of the total sample - did not allow the drawing of clear conclusions, this finding may support the need, as mentioned earlier, of using weight-adjusted doses. ${ }^{33,40}$ In addition, both studies ${ }^{24,25}$ reported significantly greater mean improvements at end point with GXR compared with placebo on both the inattention and hyperactivity/impulsivity subscales of the ADHD-RS-IV, and on secondary outcome measures, including the CGI-I, the PGA, the CPRS-R:SF and the Conners Teacher Rating Scale - revised.

An additional 9-week RCT explored the effects of GXR (1-3 mg/day, mean weight-adjusted optimal dose $0.052 \mathrm{mg} / \mathrm{kg} /$ day) on psychomotor functioning, alertness, and daytime sleepiness in 182 children and adolescents with ADHD using a laboratory-classroom setting (Table 1). ${ }^{51} \mathrm{GXR}$ was associated with a significant mean reduction in ADHDRS-IV at end point, and significantly more patients receiving GXR were rated as improved (56.8\%) compared to placebo (35.1\%). However, there were no significant differences between GXR and placebo on measures of psychomotor functioning, alertness, or other cognitive tasks; likewise, GXR was not associated with increased daytime sleepiness, thus suggesting that the beneficial effects of GXR on ADHD symptoms were independent of sedation-related effects.

The efficacy and tolerability of GXR (up to $4 \mathrm{mg} /$ day) administered in the morning (GXR-AM; mean dose $2.9 \mathrm{mg} /$ day) or in the evening (GXR-PM; mean $3 \mathrm{mg} /$ day) compared to placebo was assessed in another 8-week multicenter RCT with 333 children 6-12 years of age with ADHD (Table 1). ${ }^{52}$ GXR-AM or -PM was associated with significant and clinically meaningful improvements in ADHD symptoms, as evidenced by reductions in ADHD-RS-IV scores compared to placebo, with ESs of 0.75 for GXR-AM and 0.78 for GXR-PM, and onset of efficacy starting at first postbaseline visit. ${ }^{52}$ A post hoc analysis of the data from this dose-optimization study assessed the consistency of the effects on oppositional and ADHD symptoms throughout the day of once-daily GXR-AM or -PM monotherapy using the CPRS-R:SF. ${ }^{56}$ At end point, children taking GXR showed significantly greater improvement from baseline in mean CPRS-R:SF total score, and in each of the morning, afternoon, and evening CPRS-R:SF assessments compared with placebo, regardless of the time of GXR administration $(P<0.001)$. In addition, GXR was associated with significantly greater improvements from baseline in each CPRS$\mathrm{R}: \mathrm{SF}$ subscale score (oppositional, cognitive problems/ inattention, hyperactivity, and ADHD index) compared to placebo, regardless of time of administration, with ESs within each subscale across the different times of GXR administration ranging from 0.55 to 0.85 for GXR-AM and from 0.47 to 0.68 for GXR-PM.

More recently, Hervas et $\mathrm{al}^{49}$ assessed the efficacy and safety of once-daily dose-optimized GXR $(0.05-0.12 \mathrm{mg} / \mathrm{kg} /$ day: 6-12 years, 1-4 mg/day; 13-17 years, 1-7 mg/day) compared with placebo and ATX (10-100 mg/day) in the treatment of children and adolescents with ADHD. GXR and ATX were significantly more effective than placebo in improving core symptoms, as assessed by changes from baseline in ADHD-RS-IV total score (GXR -23.9, versus ATX -18.8, versus placebo -15.0); in addition, there was a significantly greater reduction in ADHD-RS-IV total score in GXR compared with ATX (-5.1, ES 0.440; $P=0.001)$. At end point, significantly more patients taking GXR and ATX compared to placebo showed an improvement in CGI-I (67.9\%, 56.3\%, and 44.1\% for GXR, ATX, and placebo, respectively). Statistically significant differences were seen for other key secondary variable measures, including Weiss Functional Impairment Rating Scale - parent-report learning, school, and family domains in the GXR group compared with placebo. Onset of efficacy was seen at visit 3 (week 1) for GXR and at visit 5 (week 3) for ATX.

\section{GXR for comorbid oppositional symptoms in children with ADHD}

Another placebo-controlled RCT explored the effects of GXR monotherapy (1-4 mg daily, mean $2.87 \mathrm{mg} /$ day) on oppositional symptoms in children aged 6-12 years with a diagnosis of ADHD (Table 1). ${ }^{53}$ Change scores at end point in the oppositional subscale of the CPRS-R:LF from baseline was the primary efficacy measure of this 9-week dose-optimization study. Compared with placebo, treatment with GXR was associated with significantly greater improvements in CPRS-R:LF oppositional subscale scores, with an ES of 0.59. Furthermore, GXR significantly reduced core symptoms of ADHD, as assessed by the clinician-rated ADHD-RS-IV total score, with an ES of 0.92. A post hoc correlation analysis between percentage reduction from baseline to end point in CPRS-R:LF oppositional subscale and ADHD-RS-IV total scores revealed a positive correlation 
between reductions in oppositional symptoms and ADHD core symptoms $(r=0.74)$.

In order to better characterize the effects of GXR on oppositional symptoms in children diagnosed with ADHD and comorbid ODD, a matching-adjusted indirect comparison explored the efficacy of GXR and ATX in reducing oppositional symptoms in children with this comorbidity. ${ }^{57}$ Data from 143 children with ADHD (6-12 years of age) taking GXR from the 9-week RCT evaluating the effects of GXR on comorbid oppositional symptoms ${ }^{53}$ were compared with the data from a subset analysis of 98 children aged 7-13 years taking ATX from two identical, 9-week, multisite, doubleblind RCTs assessing the safety and efficacy of ATX in ADHD and comorbid ODD ${ }^{58}$ GXR was associated with a significantly greater reduction in mean CPRS-R:LF oppositional subscale scores compared with ATX (-5.0 versus -2.4 , ES $0.58 ; P=0.01) .{ }^{57}$

\section{Efficacy of GXR for children and adolescents with ADHD in long-term studies}

Two open-label studies have assessed the long-term (up to 24 months) safety and efficacy of GXR as monotherapy or in combination with psychostimulants in the treatment of children and adolescents with ADHD. ${ }^{54,55} \mathrm{~A}$ total of 240 participants participated in the first of the extension studies, ${ }^{54}$ while 259 children $6-17$ years of age with ADHD were included in the second long-term open-label extension trial. ${ }^{55}$ Patients were recruited from the two pivotal RCTs, ${ }^{24,25}$ although the second extension trial included a small subgroup of 54 patients from a short-term open-label study of GXR adjunctive to a psychostimulant ${ }^{50}$ as well. In both studies, ${ }^{54,55}$ GXR treatment (to a maximum of $4 \mathrm{mg} /$ day) was associated with sustained, significant improvements in ADHD symptoms, as assessed by ADHD-RS-IV total score. Furthermore, both clinician-rated, based on the CGI-I, and parental-rated, as assessed with the PGA, global assessments revealed that a majority of patients - approximately $60 \%$ of the sample in either study - were much or very much improved at end point compared with baseline..$^{54,55}$

\section{Efficacy of guanfacine extended release as add-on therapy for ADHD}

GXR has also been shown to be efficacious as adjunctive therapy to psychostimulant medications, as shown in an open-label safety study ${ }^{50}$ and a large double-blind RCT. ${ }^{26}$ A multicenter, open-label, 9-week dose-escalation study examined the safety and effectiveness of GXR $(1,2,3$, or $4 \mathrm{mg} /$ day; mean $3.1 \mathrm{mg} /$ day, $0.07 \mathrm{mg} / \mathrm{kg} /$ day) administered concomitantly with MPH or AMP in 75 children and adolescents aged 6-17 years (mean age 11.6 years) with ADHD who had suboptimal control of ADHD symptoms with psychostimulants alone..$^{50}$ Coadministration of GXR and MPH or AMP was generally safe and associated with statistically and clinically significant ADHD-symptom improvement. Indeed, a significant reduction from baseline (psychostimulant alone) in ADHD-RS-IV total score was observed with combination therapy (stimulants + GXR $-16.1[56 \%], P<0.0001 ;$ MET + GXR -17.8, AMP + GXR -13.8); similar significant reductions were observed in mean scores of both the inattentiveness $(P<0.0001)$ and hyperactivity/impulsivity $(P<0.0001)$ subscales of the ADHD-RS-IV. In addition, $73 \%$ and $84.1 \%$ of children and adolescents in the study showed an improvement in CGI-I and PGA scores, respectively.

A larger 9-week, multicenter, double-blind, placebocontrolled, dose-optimization study further examined GXR (up to $4 \mathrm{mg} /$ day), either GXR-AM or GXR-PM, as an adjunct to their stable morning long-acting psychostimulant dose in a sample of 461 children and adolescents 6-17 years old (mean age 10.8 years) with suboptimal but partial response to psychostimulant alone. ${ }^{26}$ GXR-AM or -PM administered adjunctively to MPH or AMP showed significantly greater improvement over placebo plus psychostimulant in ADHD symptoms, as evidenced by reductions in ADHD-RS-IV scores (GXR-AM -4.5, GXR-PM -5.3), while ESs were 0.38 and 0.45 for the GXR-AM and GXR-PM groups, respectively. Similar significant reductions in the inattentive and the hyperactivity/impulsivity subscale ratings of the ADHD-RS-IV were seen with GXR compared to placebo, regardless of time of GXR administration. A greater percentage of patients taking GXR in combination with psychostimulants met stringent criteria for response, as assessed by $\geq 40 \%$ or $\geq 50 \%$ reduction from baseline in ADHD-RS-IV total score and remission (ADHD-RS-IV total score $\leq 18$ ) compared with placebo and psychostimulant medication. ${ }^{59}$ Significantly more subjects in both the GXR-AM and GXR-PM groups were judged as improved and to be less severely ill, as measured by CGI-I and CGI - severity scores, respectively, than subjects in the placebo group. ${ }^{26}$ In addition, participants receiving GXR along with their current stable dose of psychostimulant showed significantly greater improvement at end point on the morning and evening assessments of the Conners Global Index - Parent, as well as on the parent-rated and the participant-rated Before-School Functioning Questionnaire than participants taking placebo and psychostimulants. ${ }^{60}$ Finally, patients receiving GXR plus psychostimulant demonstrated significant reductions on the oppositional subscale of the CPRS-R:LF compared to those 
taking placebo plus psychostimulant, both in the overall study population, as well as in the subgroup of subjects with significant baseline oppositional symptoms. ${ }^{61}$

\section{Cost-effectiveness of guanfacine for ADHD}

ADHD is a costly disease associated with a large economic burden to society and the health care system. ${ }^{62,63}$ However, a growing body of research has shown that pharmacotherapy is cost-effective compared with no treatment or behavioral therapy alone among children and adolescents with ADHD. ${ }^{9,64}$ Furthermore, pharmacotherapy has been found to be cost-effective compared with combined therapy of pharmacotherapy plus behavioral treatment in the overall ADHD population. ${ }^{64}$ With the emergence of several generic formulations of certain stimulant medications and the approval of new pharmacotherapeutic alternatives for the management of ADHD, cost-effectiveness analysis of the different treatment options has become increasingly important in order to provide clinicians and payer decision makers a broader informed decision, particularly in light of the current state of limited health care resources. Recent reports have assessed the cost-effectiveness of GXR as monotherapy compared to ATX, ${ }^{65,66}$ or as an adjunctive to stimulants compared to maintaining existing stimulant monotherapy. ${ }^{67}$

The cost-effectiveness of GXR versus ATX for the treatment of ADHD in children and adolescents was explored using a novel comparative effectiveness method: matchingadjusted indirect comparison. ${ }^{65}$ The study revealed that in the trial-population GXR, within the dosing range of $0.075-0.12 \mathrm{mg} / \mathrm{kg} /$ day was more efficacious than ATX $1.2 \mathrm{mg} / \mathrm{kg} /$ day, the highest effective dose approved. ${ }^{65,66}$ In addition, the economic value of GXR as an adjunctive pharmacotherapy to stimulants compared with maintaining existing stimulant monotherapy in children and adolescents with ADHD who had a suboptimal response to stimulants alone has been assessed in a cost-effectiveness analysis, with the aim of addressing an unmet need among patients with an inadequate response to stimulant monotherapy. ${ }^{67}$ The results from this economic evaluation showed that the addition of GXR to existing stimulant monotherapy, on average, resulted in 0.028-higher quality-adjusted life-years than stimulants alone, and an incremental cost-effectiveness ratio of US\$ 31,660/quality-adjusted life-year, thus suggesting that GXR is cost-effective as an adjunctive therapy to stimulants compared with stimulant monotherapy for treating ADHD in children and adolescents with a suboptimal response to stimulant medications. ${ }^{67}$

\section{Safety and tolerability of guanfacine}

As shown in Table 2, a high proportion of patients in both the active GXR and placebo arms $-73.8 \%-84.2 \%$ and $57.7 \%-$ $75.8 \%$, respectively - experienced treatment-emergent AEs (TEAEs) in the short-term RCT of GXR for ADHD with a cumulative sample of 1,617 children and adolescents (GXR 1,107, placebo 510). ${ }^{24,25,49,51-53}$ The most common TEAEs in these studies associated with GXR compared with placebo were somnolence $(27.0 \%-50.7 \%$ versus $3.5 \%-22.8 \%)$, headache $(16.7 \%-26.3 \%$ versus $10.6 \%-24.3 \%)$, upper abdominal pain $(6.1 \%-14.3 \%$ versus $2.6 \%-9.1 \%)$, fatigue (10.9\%-25.4\% versus $2.0 \%-18.0 \%)$, and sedation $(5.9 \%-$ $14.5 \%$ versus $2.7 \%-4.5 \%$ ). Similar rates of TEAEs were detected in long-term extension studies of GXR for ADHD, with somnolence, headache, fatigue, and upper abdominal pain the most common TEAEs. ${ }^{54,55}$ Likewise, a systematic review and meta-analysis of published and unpublished studies reported that compared with placebo, guanfacine was safe and generally well tolerated. ${ }^{28} \mathrm{~A}$ total of 948 participants (82.4\%) receiving guanfacine and 376 (67.9\%) taking placebo experienced at least one TEAE (OR 2.6), somnolence (OR 4.9), sedation (OR 2.8), and fatigue (OR 2.2) the most common TEAEs. ${ }^{28}$ Many of these adverse reactions, particularly sedation-related TEAEs, were dose-related, transient, mild to moderate in severity, and did not interfere with attention. ${ }^{22,27,41}$ Furthermore, as described earlier, GXR efficacy is seemingly independent of general sedationrelated effects. ${ }^{51}$

The incidence and severity of TEAEs with adjunctive administration of GXR to a psychostimulant ${ }^{26,50}$ was overall similar to that observed in the clinical studies of GXR alone. Moreover, no unique TEAE emerged from GXR adjunctive to a long-acting psychostimulant. ${ }^{26,50}$ This is consistent with findings from studies conducted in healthy adults showing that no unique TEAEs were observed with coadministration of GXR and $\mathrm{MPH}^{47}$ or lisdexamfetamine ${ }^{48}$ compared with either treatment alone.

As expected, discontinuation rates due to TEAEs in the clinic were higher in the groups of children and adolescents receiving GXR than in those on placebo. Indeed, $3.3 \%-16.2 \%$ of patients in the active GXR groups compared to $0 \%-7.6 \%$ in the placebo groups discontinued the study because of TEAEs in short-term RCTs..$^{24,25,49,51-53}$ Likewise, the incidence of discontinuation as a result of TEAEs in studies evaluating the safety and efficacy of GXR as an adjunctive treatment to stimulant medications was $6.6 \%-6.7 \%$ for individuals taking GXR and stimulants ${ }^{26,50}$ and $0.7 \%$ for placebo and stimulants. ${ }^{26}$ In the two long-term extension 


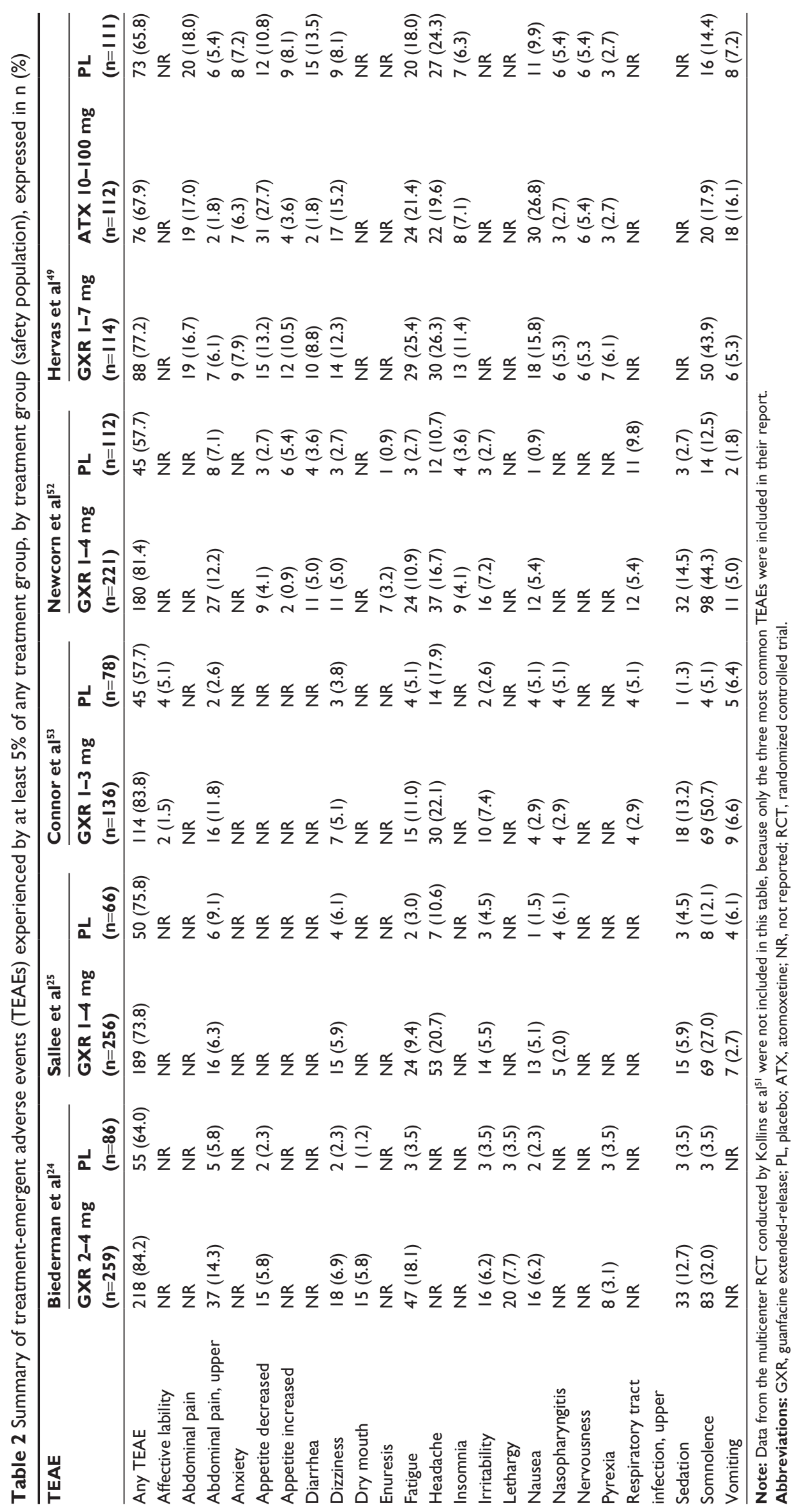


studies, discontinuation rates of GXR as a consequence of TEAEs were $13.5 \%{ }^{55}$ and $26.9 \% .^{54}$

\section{Cardiovascular safety of GXR}

Dose-dependent, small-to-modest reductions in systolic BP (SBP), diastolic BP (DBP), and HR have been observed in GXR-treated patients, as monotherapy or as an addon to psychostimulants, compared to placebo, approximately $-5 \mathrm{mmHg}$ (SBP), $-3 \mathrm{mmHg}$ (DBP), and $-6 \mathrm{bpm}$ (HR) for all dose groups combined. ${ }^{24-26,51}$ Following longterm treatment with guanfacine, as with other $\alpha_{2}$-adrenergic agonists, tolerance to the hypotensive effects develops, and abrupt withdrawal-rebound hypertension for several weeks may occur. 22,33,68 Therefore, when tapering off GXR, gradual dose reduction of no more than $1 \mathrm{mg}$ every 3-7 days is recommended. ${ }^{33}$

There are no reports of serious cardiovascular AEs, including no serious QTc abnormalities, associated with GXR alone or in combination with psychostimulants in the different multisite studies. ${ }^{27,69}$ Across the different short- and long-term studies assessing the efficacy and safety of GXR for ADHD, minor changes in electrocardiography (ECG) parameters apparently lacking clinical significance have been reported, with no patient presenting a QRS interval $\geq 120 \mathrm{~ms}$, QTc interval $\geq 500 \mathrm{~ms}$ (using Fridericia's correction formula, correction QTcF), or QTcF increase from baseline $\geq 60$ ms. ${ }^{24,25,50,51,53,54}$ Mean increases in QTcF from baseline in GXR-treated patients across the different RCTs ranged from $6.1-9.1 \mathrm{~ms},{ }^{24} 2.4-9.7 \mathrm{~ms},{ }^{25} 3.3 \mathrm{~ms},{ }^{50}$ and $5.2 \mathrm{~ms},{ }^{53}$ to $-0.1 \mathrm{~ms}^{54}$ where detailed QTcF reporting was provided. No "meaningful difference" in QTc intervals were reported in four studies, ${ }^{26,49,51,55}$ and one RCT did not assess QTc intervals. ${ }^{52}$ Similarly, there are no reports of serious cardiovascular AEs when combining GXR and psychostimulant medication. Changes from baseline in QTcF intervals ranged in one of the studies $^{50}$ from 0.01 to $5.15 \mathrm{~ms}$, and no subject met the ECGoutlier criteria (PR interval $\geq 200 \mathrm{~ms}$, QRS interval $\geq 120 \mathrm{~ms}$, QTc interval $\geq 500 \mathrm{~ms}$, or QTc change from baseline $\geq 60 \mathrm{~ms}$ ). In a large multicenter RCT, ${ }^{26}$ no subject, regardless of dose and time of administration, developed a QTc $\geq 480 \mathrm{~ms}$ (using Fridericia's formula) or QT $\geq 500 \mathrm{~ms}$ (using Bazett's formula). These findings from the different trials assessing the efficacy of GXR for ADHD are consistent with a thorough, double-blind, crossover QT study of GIR (at therapeutic [4 mg] and supratherapeutic [8 $\mathrm{mg}$ ] doses) conducted in a sample of 83 healthy adults. ${ }^{70}$ Moreover, as expected, TEAEs were mild, but neither therapeutic nor supratherapeutic doses of guanfacine prolonged QTcF interval through 12 hours postdose, thus supporting the view that guanfacine does not appear to interfere with cardiac repolarization of the form associated with proarrhythmic drugs. ${ }^{70}$

\section{Conclusion}

As evidenced in short-term RCTs and in long-term open-label extension studies, GXR administered once daily either in the morning or evening has been shown to be effective as monotherapy in children and adolescents with ADHD. ${ }^{24,25,28,49,51-55}$ Furthermore, GXR has also been associated with significant and clinically meaningful improvements in ADHD symptoms as adjunctive therapy to MPH- or AMP-based medications in patients with suboptimal responses to stimulants. ${ }^{26,50}$ Indeed, the treatment ESs across the different studies are comparable with that observed with other medications used for treating children and adolescents with ADHD. ${ }^{23,71}$ Moreover, GXR may show greater symptom reduction than ATX. ${ }^{49,57}$ The effectiveness of GXR on oppositional symptoms in children with $\mathrm{ADHD}^{53,56,57}$ could be of particular interest, based on the high rates of comorbid ODD and ADHD, ${ }^{8}$ but also considering that atypical antipsychotics are widely used, generally in combination, for patients with ODD or other severe comorbidities. ${ }^{72}$ In contrast, the different studies demonstrated a positive risk-benefit profile of GXR in the treatment of children and adolescents with ADHD. ${ }^{24-26,28,49-51,53-55}$ While the incidence of sedation-related AEs is more frequent than with ATX or stimulants, ${ }^{51}$ these appear to be unrelated to the therapeutic effects of GXR, and the overall pattern and incidence of TEAEs are consistent with that seen with other medications approved for ADHD. ${ }^{73}$ Although BP and HR should be monitored prior to initiation of therapy and periodically during guanfacine treatment, ECG monitoring is unnecessary unless there is a personal or family history of cardiac disease, arrhythmias, or sudden unexpected death. ${ }^{68,69}$ Overall, the reviewed evidence suggests that GXR will be a useful and safe nonstimulant treatment option for the broadspectrum management of ADHD symptoms.

\section{Disclosure}

JM-R has received honoraria for being a speaker and on advisory boards from Shire and Janssen Pharmaceuticals. $\mathrm{CK}$ and $\mathrm{RdA}$ report no conflicts of interest in this work. No sources of funding were used in the preparation, review, or approval of this manuscript.

\section{References}

1. American Psychiatric Association. Diagnostic and Statistical Manual of Mental Disorders. 5th ed. Arlington (VA): American Psychiatric Publishing; 2013.

2. Feldman HM, Reiff MI. Clinical practice. Attention deficit-hyperactivity disorder in children and adolescents. N Engl J Med. 2014;370(9): 838-846. 
3. Polanczyk GV, Willcutt EG, Salum GA, Kieling C, Rohde LA. ADHD prevalence estimates across three decades: an updated systematic review and meta-regression analysis. Int J Epidemiol. 2014;43(2):434-442.

4. Willcutt EG. The prevalence of DSM-IV attention-deficit/hyperactivity disorder: a meta-analytic review. Neurotherapeutics. 2012;9(3): 490-499.

5. Gau SS, Ni HC, Shang CY, et al. Psychiatric comorbidity among children and adolescents with and without persistent attention-deficit hyperactivity disorder. Aust N Z J Psychiatry. 2010;44(2):135-143.

6. Kooij JJ, Huss M, Asherson P, et al. Distinguishing comorbidity and successful management of adult ADHD. J Atten Disord. 2012;16 (5 Suppl):3S-19S.

7. Martinez-Raga J, Szerman N, Knecht C, de Alvaro R. Attention deficit hyperactivity disorder and dual disorders. Educational needs for an underdiagnosed condition. Int J Adolesc Med Health. 2013;25(3): 231-243.

8. Connor DF, Doerfler LA. ADHD with comorbid oppositional defiant disorder or conduct disorder: discrete or non-distinct disruptive behavior disorders? J Atten Disord. 2008;12(2):126-134.

9. National Collaborating Centre for Mental Health. Attention Deficit Hyperactivity Disorder: The NICE Guideline on Diagnosis and Management of ADHD in Children, Young People and Adults. London: Alden Press; 2009.

10. Akutagava-Martins GC, Salatino-Oliveira A, Kieling CC, Rohde LA, Hutz MH. Genetics of attention-deficit/hyperactivity disorder: current findings and future directions. Expert Rev Neurother. 2013;13(4): 435-445.

11. Freitag CM, Rohde LA, Lempp T, Romanos M. Phenotypic and measurement influences on heritability estimates in childhood ADHD. Eur Child Adolesc Psychiatry. 2010;19(3):311-323.

12. Cortese S, Kelly C, Chabernaud C, et al. Toward systems neuroscience of ADHD: a meta-analysis of $55 \mathrm{fMRI}$ studies. Am J Psychiatry. 2012;169(10):1038-1055.

13. Arnsten AF. The emerging neurobiology of attention deficit hyperactivity disorder: the key role of the prefrontal association cortex. J Pediatr. 2009;154(5):I-S43.

14. Stahl SM. The prefrontal cortex is out of tune in attention-deficit/ hyperactivity disorder. J Clin Psychiatry. 2009;70(7):950-951.

15. Bradley C. The behavior of children receiving Benzedrine. Am J Psychiatry. 1937;94(3):577-585.

16. Canadian Attention Deficit Hyperactivity Disorder Resource Alliance (CADDRA). Canadian ADHD Practice Guidelines. 3rd ed. Toronto: CADDRA; 2011.

17. Kooij SJ, Bejerot S, Blackwell A, et al. European consensus statement on diagnosis and treatment of adult ADHD: the European Network Adult ADHD. BMC Psychiatry. 2010;10:67.

18. Subcommittee on Attention-Deficit Hyperactivity. ADHD: clinical practice guideline for the diagnosis, evaluation, and treatment of attention-deficit/hyperactivity disorder in children and adolescents. Pediatrics. 2011;128(5):1007-1022.

19. Biederman J, Faraone SV. Attention-deficit hyperactivity disorder. Lancet. 2005;366(9481):237-248.

20. Peterson K, McDonagh MS, Fu R. Comparative benefits and harms of competing medications for adults with attention-deficit hyperactivity disorder: a systematic review and indirect comparison meta-analysis. Psychopharmacology (Berl). 2008;197(1):1-11.

21. Cortese S, Holtmann M, Banaschewski T, et al. Practitioner review: current best practice in the management of adverse events during treatment with ADHD medications in children and adolescents. J Child Psychol Psychiatry. 2013;54(3):227-246.

22. Faraone SV, McBurnett K, Sallee FR, Steeber J, López FA. Guanfacine extended release: a novel treatment for attention-deficit/ hyperactivity disorder in children and adolescents. Clin Ther. 2013; 35(11):1778-1793.

23. Savill NC, Buitelaar JK, Anand E, et al. The efficacy of atomoxetine for the treatment of children and adolescents with attention-deficit/ hyperactivity disorder: a comprehensive review of over a decade of clinical research. CNS Drugs. 2015;29(2):131-151.
24. Biederman J, Melmed RD, Patel A, et al. A randomized, double-blind, placebo-controlled study of guanfacine extended release in children and adolescents with attention-deficit/hyperactivity disorder. Pediatrics. 2008;121(1):e73-e84.

25. Sallee FR, McGough J, Wigal T, Donahue J, Lyne A, Biederman J. Guanfacine extended release in children and adolescents with attentiondeficit/hyperactivity disorder: a placebo-controlled trial. $J$ Am Acad Child Adolesc Psychiatry. 2009;48(2):155-165.

26. Wilens TE, Bukstein O, Brams M, et al. A controlled trial of extendedrelease guanfacine and psychostimulants for attention-deficit/ hyperactivity disorder. J Am Acad Child Adolesc Psychiatry. 2012;51(1): 74-85

27. Elbe D, Reddy D. Focus on guanfacine extended-release: a review of its use in child and adolescent psychiatry. $J$ Can Acad Child Adolesc Psychiatry. 2014;23(1):48-60.

28. Ruggiero S, Clavenna A, Reale L, Capuano A, Rossi F, Bonati M. Guanfacine for attention deficit and hyperactivity disorder in pediatrics: a systematic review and meta-analysis. Eur Neuropsychopharmacol. 2014;24(10):1578-1590.

29. Cohen DJ, Young JG, Nathanson JA, Shaywitz BA. Clonidine in Tourette's syndrome. Lancet. 1979;2(8142):551-553.

30. Gourlay S, Forbes A, Marriner T, Kutin J, McNeil J. A placebocontrolled study of three clonidine doses for smoking cessation. Clin Pharmacol Ther. 1994;55(1):64-69.

31. Gowing LR, Farrell M, Ali RL, White JM. Alpha2-adrenergic agonists for the management of opioid withdrawal. Cochrane Database Syst Rev. 2014;3:CD002024.

32. Sallee F, Connor DF, Newcorn JH. A review of the rationale and clinical utilization of $\alpha 2$-adrenoceptor agonists for the treatment of attention-deficit/hyperactivity and related disorders. $J$ Child Adolesc Psychopharmacol. 2013;23(5):308-319.

33. Intuniv (guanfacine extended-release tablets) [US prescribing information]. Wayne (PA): Shire Pharmaceuticals Inc; 2014.

34. Jain R, Segal S, Kollins SH, Khayrallah M. Clonidine extended-release tablets for pediatric patients with attention-deficit/hyperactivity disorder. J Am Acad Child Adolesc Psychiatry. 2011;50(2):171-179.

35. Bidwell LC, Dew RE, Kollins SH. Alpha-2 adrenergic receptors and attention-deficit/hyperactivity disorder. Curr Psychiatry Rep. 2010;12(5):366-373.

36. Scahill L. Alpha-2 adrenergic agonists in children with inattention, hyperactivity and impulsiveness. CNS Drugs. 2009;23 Suppl 1: 43-49.

37. Arnsten AF, Scahill L, Findling RL. Alpha2-adrenergic receptor agonists for the treatment of attention-deficit/hyperactivity disorder: emerging concepts from new data. J Child Adolesc Psychopharmacol. 2007;17(4):393-406.

38. Connor DF, Rubin J. Guanfacine extended release in the treatment of attention deficit hyperactivity disorder in children and adolescents. Drugs Today (Barc). 2010;46(5):299-314.

39. Wang M, Ramos BP, Paspalas CD, et al. Alpha2A-adrenoceptors strengthen working memory networks by inhibiting cAMP-HCN channel signaling in prefrontal cortex. Cell. 2007;129(2):397-410.

40. Boellner SW, Pennick M, Fiske K, Lyne A, Shojaei A. Pharmacokinetics of a guanfacine extended-release formulation in children and adolescents with attention-deficit-hyperactivity disorder. Pharmacotherapy. 2007;27(9):1253-1262.

41. Bukstein OG, Head J. Guanfacine ER for the treatment of adolescent attention-deficit/hyperactivity disorder. Expert Opin Pharmacother. 2012;13(15):2207-2213.

42. Kiechel J. Pharmacokinetics and metabolism of guanfacine in man: a review. Br J Clin Pharmacol. 1980;10 Suppl 1:25S-32S.

43. Swearingen D, Pennick M, Shojaei A, Lyne A, Fiske K. A phase I, randomized, open-label, crossover study of the single-dose pharmacokinetic properties of guanfacine extended-release 1-, 2-, and 4-mg tablets in healthy adults. Clin Ther. 2007;29(4):617-625.

44. Gillis NK, Zhu HJ, Markowitz JS. An in vitro evaluation of guanfacine as a substrate for P-glycoprotein. Neuropsychiatr Dis Treat. 2010;7(1):501-505. 
45. Muir VJ, Perry CM. Guanfacine extended-release: in attention deficit hyperactivity disorder. Drugs. 2010;70(13):1693-1702.

46. Ambrosini PJ, Sheikh M. Increased plasma valproate concentrations when coadministered with guanfacine. J Child Adolesc Psychopharmacol. 1998;8(2):143-147.

47. Roesch B, Corcoran M, Haffey M, et al. Pharmacokinetics of coadministration of guanfacine extended release and methylphenidate extended release. Drugs R D. 2013;13(1):53-61.

48. Roesch B, Corcoran ME, Fetterolf J, et al. Pharmacokinetics of coadministered guanfacine extended release and lisdexamfetamine dimesylate. Drugs R D. 2013;13(2):119-128.

49. Hervas A, Huss M, Johnson M, et al. Efficacy and safety of extendedrelease guanfacine hydrochloride in children and adolescents with attention-deficit/hyperactivity disorder: a randomized, controlled, phase III trial. Eur Neuropsychopharmacol. 2014;24(12):1861-1872.

50. Spencer TJ, Greenbaum M, Ginsberg LD, Murphy WR. Safety and effectiveness of coadministration of guanfacine extended release and psychostimulants in children and adolescents with attentiondeficit/hyperactivity disorder. J Child Adolesc Psychopharmacol. 2009;19(5):501-510.

51. Kollins SH, López FA, Vince BD, et al. Psychomotor functioning and alertness with guanfacine extended release in subjects with attentiondeficit/hyperactivity disorder. J Child Adolesc Psychopharmacol. 2011;21(2):111-120.

52. Newcorn JH, Stein MA, Childress AC, et al. Randomized, double-blind trial of guanfacine extended release in children with attention-deficit/ hyperactivity disorder: morning or evening administration. $J$ Am Acad Child Adolesc Psychiatry. 2013;52(9):921-930.

53. Connor DF, Findling RL, Kollins SH, et al. Effects of guanfacine extended release on oppositional symptoms in children aged 6-12 years with attention-deficit hyperactivity disorder and oppositional symptoms: a randomized, double-blind, placebo-controlled trial. CNS Drugs. 2010;24(9):755-768.

54. Biederman J, Melmed RD, Patel A, McBurnett K, Donahue J, Lyne A. Long-term, open-label extension study of guanfacine extended release in children and adolescents with ADHD. CNS Spectr. 2008;13(12): 1047-1055.

55. Sallee FR, Lyne A, Wigal T, Mcgough JJ. Long-term safety and efficacy of guanfacine extended release in children and adolescents with attention-deficit/hyperactivity disorder. J Child Adolesc Psychopharmacol. 2009;19(3):215-226.

56. Young J, Rugino T, Dammerman R, Lyne A, Newcorn JH. Efficacy of guanfacine extended release assessed during the morning, afternoon, and evening using a modified Conners' parent rating scale-revised: short form. J Child Adolesc Psychopharmacol. 2014;24(8):435-441.

57. Signorovitch J, Erder MH, Xie J, et al. Comparative effectiveness research using matching-adjusted indirect comparison: an application to treatment with guanfacine extended release or atomoxetine in children with attention-deficit/hyperactivity disorder and comorbid oppositional defiant disorder. Pharmacoepidemiol Drug Saf. 2012; 21 Suppl 2:130-137.

58. Kaplan S, Heiligenstein J, West S, et al. Efficacy and safety of atomoxetine in childhood attention-deficit/hyperactivity disorder with comorbid oppositional defiant disorder. J Atten Disord. 2004;8(2):45-52.
59. Cutler AJ, Brams M, Bukstein O, et al. Response/remission with guanfacine extended-release and psychostimulants in children and adolescents with attention-deficit/hyperactivity disorder. J Am Acad Child Adolesc Psychiatry. 2014;53(10):1092-1101.

60. Wilens TE, McBurnett K, Turnbow J, Rugino T, White C, Youcha S. Morning and evening effects of guanfacine extended release adjunctive to psychostimulants in pediatric ADHD: results from a phase III multicenter trial. J Atten Disord. Epub 2013 Sep 26.

61. Findling RL, McBurnett K, White C, Youcha S. Guanfacine extended release adjunctive to a psychostimulant in the treatment of comorbid oppositional symptoms in children and adolescents with attentiondeficit/hyperactivity disorder. J Child Adolesc Psychopharmacol. 2014;24(5):245-252.

62. Doshi JA, Hodgkins P, Kahle J, et al. Economic impact of childhood and adult attention-deficit/hyperactivity disorder in the United States. J Am Acad Child Adolesc Psychiatry. 2012;51(10):990-1002.

63. Wittchen HU, Jacobi F, Rehm J, et al. The size and burden of mental disorders and other disorders of the brain in Europe 2010. Eur Neuropsychopharmacol. 2011;21(9):655-679.

64. Wu EQ, Hodgkins P, Ben-Hamadi R, et al. Cost effectiveness of pharmacotherapies for attention-deficit hyperactivity disorder: a systematic literature review. CNS Drugs. 2012;26(7):581-600.

65. Erder MH, Xie J, Signorovitch JE, et al. Cost effectiveness of guanfacine extended-release versus atomoxetine for the treatment of attentiondeficit/hyperactivity disorder application of a matching-adjusted indirect comparison. Appl Health Econ Health Policy. 2013;10(6):381-395.

66. Sikirica V, Findling RL, Signorovitch J, et al. Comparative efficacy of guanfacine extended release versus atomoxetine for the treatment of attention-deficit/hyperactivity disorder in children and adolescents: applying matching-adjusted indirect comparison methodology. CNS Drugs. 2013;27(11):943-953.

67. Sikirica V, Erder MH, Xie J, et al. Cost effectiveness of guanfacine extended release as an adjunctive therapy to a stimulant compared with stimulant monotherapy for the treatment of attention-deficit hyperactivity disorder in children and adolescents. Pharmacoeconomics. 2012;30(8):e1-e15.

68. Vitiello B, Towbin K. Stimulant treatment of ADHD and risk of sudden death in children. Am J Psychiatry. 2009;166(9):955-957.

69. Martinez-Raga J, Knecht C, Szerman N, Martinez MI. Risk of serious cardiovascular problems with medications for attention-deficit hyperactivity disorder. CNS Drugs. 2013;27(1):15-30.

70. Martin P, Satin L, Kahn RS, et al. A thorough QT study of guanfacine. Int J Clin Pharmacol Ther. 2015;53(4):301-316.

71. Faraone SV, Buitelaar J. Comparing the efficacy of stimulants for ADHD in children and adolescents using meta-analysis. Eur Child Adolesc Psychiatry. 2010;19(4):353-364.

72. Weiss M, Panagiotopoulos C, Giles L, et al. A naturalistic study of predictors and risks of atypical antipsychotic use in an attentiondeficit/hyperactivity disorder clinic. J Child Adolesc Psychopharmacol. 2009;19(5):575-582.

73. Graham J, Coghill D. Adverse effects of pharmacotherapies for attention-deficit hyperactivity disorder. Epidemiology, prevention and management. CNS Drugs. 2008;22(3):213-237.
Neuropsychiatric Disease and Treatment

\section{Publish your work in this journal}

Neuropsychiatric Disease and Treatment is an international, peerreviewed journal of clinical therapeutics and pharmacology focusing on concise rapid reporting of clinical or pre-clinical studies on a range of neuropsychiatric and neurological disorders. This journal is indexed on PubMed Central, the 'PsycINFO' database and CAS,

\section{Dovepress}

and is the official journal of The International Neuropsychiatric Association (INA). The manuscript management system is completely online and includes a very quick and fair peer-review system, which is all easy to use. Visit http://www.dovepress.com/testimonials.php to read real quotes from published authors. 